BRX-TH 6648

CALT-TH 2019-008

UFIFT-QG-19-02

\title{
Nonlocal Cosmology II - Cosmic acceleration without fine tuning or dark energy
}

\author{
S. Deser ${ }^{1 *}$ and R. P. Woodard ${ }^{2 *}$ \\ ${ }^{1}$ California Institute of Technology, Pasadena, CA 91125 and \\ Department of Physics, Brandeis University, Waltham, MA 02254 \\ ${ }^{2}$ Department of Physics, University of Florida, \\ Gainesville, FL 32611
}

\begin{abstract}
We present an improved version of our original cosmological model to explain the current phase of cosmological acceleration without resorting to a cosmological constant or any other mass scale. Like the original, this phenomenological approach is based on an effective quantum gravitational action, but now depends on the original nonlocal dimensionless scalar $X=\mathbf{\square}^{-1} R$ only through $Y=\mathbf{\square}^{-1} g^{\mu \nu} X_{, \mu} X_{, \nu}$. Both $X$ and $Y$ are quiescent during the radiation-dominated $(R=0)$ era, both only grow logarithmically during matter dominance, and neither affects the propagation of gravitational radiation. However, while $X$ has the same sign for gravitationally bound systems as for cosmology, we show that the sign of $Y$ differs for the two cases: it is positive for cosmology and negative for gravitationally bound systems. We can therefore enforce the $\Lambda \mathrm{CDM}$ expansion history by making a suitable choice of the nonlocal distortion function $f(Y)$ for $Y>0$, while ensuring that there is no change in the heavily constrained phenomenology of gravitationally bound systems simply by making $f$ vanish for $Y<0$. We numerically determine the required function $f(Y>0)$ : it has a surprisingly simple exponential form.
\end{abstract}

PACS numbers: 04.50.Kd, 95.35.+d, 98.62.-g

* e-mail: deser@brandeis.edu,woodard@phys.ufl.edu 


\section{Introduction}

This work is a continuation, and shares the basis and philosophy, of our original cosmological model [1 3]. The present cosmological acceleration phase of the universe [4] is a major, if originally unexpected, feature of late time expansion. An explanation not invoking new physics or fine tuning is clearly to be preferred; ours was a nonlocal one, based on a function of the dimensionless scalar $X[g]=\square^{-1} R$. The argument was that it represents current effects of the necessarily abundant infrared gravitons in the early universe [5, 6].

Extensive studies have been made of the theory's cosmological perturbations [7-12]. There have also been studies of future cosmological evolution [13], solar system constraints [14], and the generation of gravitational radiation [15] 1 ]

Our original model assumed that $X[g]$ had opposite signs in the cosmological $(-)$ and the (smaller scale) gravitationally bound $(+)$ contexts. That would prevent - unwanted - effects in the latter. However, it was recently pointed out that $X[g]$ is negative definite [24]. We overcome this difficulty by a simple modification: replacing $X[g]$ by the (equally nonlocal) $Y[g] \equiv \square^{-1}\left[g^{\mu \nu} \partial_{\mu} X \partial_{\nu} X\right]$, removes the problem without losing the explanation of accelerated expansion: While both $X[g]$ and $Y[g]$ vanish during radiation domination $(R=0)$, and only grow slowly thereafter, $Y[g]$ does have opposite signs in bound matter $(Y<0)$ and in the large $(Y>0)$; so we merely define the nonlocal distortion function $f(Y)$ to vanish for $Y<0$, and have the proper details for $Y>0$, thus restoring the desired behavior throughout. An additional, highly desirable property of both the original and the new theories is that there is no change in the constrained propagation of gravitational radiation [25].

Section 2 defines our model and discusses how it might emerge from fundamental considerations. It also explains why the new nonlocal scalar $Y[g]$ changes sign from cosmological to gravitationally bound systems. Section 3 gives an explicit numerical determination of the nonlocal distortion function $f(Y)$ to reproduce the $\Lambda$ CDM expansion history without a cosmological constant. It also derives an amazingly simple exponential fit to $f(Y)$. Section 4 presents conclusions.

\footnotetext{
${ }^{1} \mathrm{~A}$ similar model has also been proposed which is based on the dimensionful nonlocal scalar $\square^{-2} R$ [16, 17. Many studies have been made of the phenomenology of this model [18 23].
} 


\section{The New Model}

In this section, we define and discuss the improved model. The original problem and its remedy are explained. We close with comments on its possible origin in a more fundamental setting.

\subsection{Defining the New Model}

Our two nonlocal scalars are

$$
X[g] \equiv \frac{1}{\square} R, Y[g] \equiv \frac{1}{\square}\left(g^{\mu \nu} \partial_{\mu} X[g] \partial_{\nu} X[g]\right) ; \square \equiv \frac{1}{\sqrt{-g}} \partial_{\mu}\left(\sqrt{-g} g^{\mu \nu} \partial_{\nu}\right),
$$

where $\boldsymbol{\square}^{-1}$ is defined by retarded boundary conditions which require that $X[g](x), Y[g](x)$ and their first derivatives all vanish on the initial value surface. Our nonlocal modification consists of adding $R$ times a distortion function $f(Y)$,

$$
\mathcal{L}_{\text {nonlocal }} \equiv \frac{1}{16 \pi G} R[1+f(Y[g])] \sqrt{-g} .
$$

Just as the original model could be localized through the introduction of two auxiliary scalar fields [26], the new model requires four auxiliaries, 2 ]

$$
\mathcal{L}_{\text {local }} \equiv \frac{\sqrt{-g}}{16 \pi G}\left[R(1+U+f(Y))+\left(\partial_{\mu} X \partial_{\nu} U+\partial_{\mu} Y \partial_{\nu} V+V \partial_{\mu} X \partial_{\nu} X\right) g^{\mu \nu}\right]
$$

It is important to bear in mind that the auxiliary scalars do not have arbitrary initial value data, which would result in new degrees of freedom, half being ghosts [3, 27]. Instead, all obey retarded boundary conditions, hence introduce no excitations.

The $U, V$ Lagrange multipliers, whose variations lead to the equations which, with retarded boundary conditions, define $X[g]$ and $Y[g]$,

$$
\begin{aligned}
& \frac{16 \pi G}{\sqrt{-g}} \frac{\delta S}{\delta U}=-\square X+R=0 \quad \Longrightarrow \quad X[g]=\frac{1}{\square} R, \\
& \frac{16 \pi G}{\sqrt{-g}} \frac{\delta S}{\delta V}=-\square Y+g^{\mu \nu} X_{, \mu} X_{, \nu}=0 \quad \Longrightarrow \quad Y[g]=\frac{1}{\square}\left[g^{\mu \nu} X_{, \mu} X_{, \nu}\right] .
\end{aligned}
$$

\footnotetext{
${ }^{2}$ We shall abuse the notation slightly by using the same symbols $X$ and $Y$ for auxiliary scalars in the localized model (3) as for their retarded solutions (11).
} 
Variation with respect to $X$ and $Y$ leads to similar equations for $U$ and $V$, which are also solved with retarded boundary conditions,

$$
\begin{aligned}
& \frac{16 \pi G}{\sqrt{-g}} \frac{\delta S}{\delta X}=-\square U-2 D_{\mu}\left(V D^{\mu} X\right)=0 \quad \Longrightarrow \quad U[g]=-\frac{2}{\square} D_{\mu}\left(V D^{\mu} X\right), \\
& \frac{16 \pi G}{\sqrt{-g}} \frac{\delta S}{\delta Y}=-\square V+R f^{\prime}(Y)=0 \quad \Longrightarrow \quad V[g]=\frac{1}{\square} R f^{\prime}(Y) .
\end{aligned}
$$

The gravitational field equations are,

$$
\begin{aligned}
& \left(G_{\mu \nu}+g_{\mu \nu} \mathbf{\square}-D_{\mu} D_{\nu}\right)(1+U+f(Y))+\partial_{(\mu} X \partial_{\nu)} U+\partial_{(\mu} Y \partial_{\nu)} V \\
& \quad+V \partial_{\mu} X \partial_{\nu} X-\frac{1}{2} g_{\mu \nu} g^{\rho \sigma}\left(\partial_{\rho} X \partial_{\sigma} U+\partial_{\rho} Y \partial_{\sigma} V+V \partial_{\rho} X \partial_{\sigma} X\right)=8 \pi G T_{\mu \nu}
\end{aligned}
$$

here parenthesized indices are symmetrized and $T_{\mu \nu}$ is the matter stressenergy tensor without dark energy.

\subsection{The signs of $Y$}

To determine the sign of $Y$ in regions of bound matter, we assume the metric there to be (quasi-) static, i.e., time-independent and diagonal; it could more generally be stationary, $g_{0 i} \neq 0$, but the same considerations should still hold with a bit more matrix detail. Then $g^{\mu \nu} X_{, \mu} X_{, \nu} \rightarrow g^{i j} X_{, i} X_{, j}$ is positive in our $(-+++)$ convention. We argue next that, instead, $\square^{-1}$ is negative so that there $Y<0$ also . Recall that in flat space, $\square^{-1}$ acting on a time independent source reduces, upon time-integration, to $\nabla^{-2}$. But our $\square$ has the flat-space $-\partial_{t}^{2}$ form, namely $-g^{00}\left[-g_{00} \nabla^{2}-\partial_{t}^{2}\right]$, except for the overall $-g^{00}$ and the (irrelevant) metric dependence of our $\nabla^{2}$; there is also a (strictly positive) factor $\sqrt{-g}=\sqrt{{ }^{3} g} \sqrt{-g_{00}}$ upstairs. Thus, after time integration, our net inverse Laplacian is $\frac{1}{\nabla^{2}}$ (since $g^{00} g_{00}=1$ ), a negative quantity when operating on the positive $g^{i j} X_{, i} X_{, j}$. In section 3 we show, by explicit computation, that $Y$ is positive in the purely time dependent cosmological region.

Another, global, way of understanding the signs of both $X$ and $Y$ is by taking the flat space limit. The retarded Green's function $G_{\mathrm{ret}}[g]\left(x ; x^{\prime}\right)$ which implements $\square^{-1}$ reduces, when $g_{\mu \nu} \rightarrow \eta_{\mu \nu}$, to the usual flat

$$
G_{\mathrm{ret}}[\eta]\left(x ; x^{\prime}\right)=-\frac{\delta\left(t-t^{\prime}-\frac{1}{c}\left\|\vec{x}-\vec{x}^{\prime}\right\|\right)}{4 \pi\left\|\vec{x}-\vec{x}^{\prime}\right\|} .
$$


This simple form makes it easy to derive explicit expressions for $X$ and $Y$. For example, if the Ricci scalar is a positive constant and the initial value surface is at $t=0$,

$$
R(x)=\frac{1}{\ell^{2}} \quad \Longrightarrow \quad X(x)=-\frac{c^{2} t^{2}}{2 \ell^{2}} \quad, \quad Y(x)=+\frac{c^{4} t^{4}}{12 \ell^{4}} .
$$

This situation in which the time dependence of $X$ dominates is relevant to cosmology. On the other hand, suppose the Ricci scalar is a positive constant within a sphere of radius $\ell$, and we consider some time $t$ much larger than either $\ell$ or $\|\vec{x}\| \equiv r$,

$$
R(x)=\frac{\theta(\ell-\|\vec{x}\|)}{\ell^{2}} \Longrightarrow X(x)=-\frac{1}{3}\left[\frac{3}{2}-\frac{r^{2}}{2 \ell^{2}}\right] \theta(\ell-r)-\frac{\ell}{3 r} \theta(r-\ell) .
$$

The result for $X$ is still negative definite, but the space derivatives dominate,

$$
R(x)=\frac{\theta(\ell-\|\vec{x}\|)}{\ell^{2}} \Longrightarrow g^{\mu \nu} \partial_{\mu} X \partial_{\nu} X=\left(\frac{\partial X}{\partial r}\right)^{2}=\frac{r^{2}}{9 \ell^{4}} \theta(\ell-r)+\frac{\ell^{2}}{9 r^{4}} \theta(r-\ell) .
$$

That reverses the sign of $Y$ from the cosmological case (10),

$R(x)=\frac{\theta(\ell-\|\vec{x}\|)}{\ell^{2}} \Longrightarrow Y(x)=-\frac{1}{18}\left[\frac{3}{2}-\frac{r^{4}}{10 \ell^{4}}\right] \theta(\ell-r)-\frac{1}{18}\left[\frac{12 \ell}{5 r}-\frac{\ell^{2}}{r^{2}}\right] \theta(r-\ell)$

\subsection{Connection to Fundamentals}

We do not believe that nonlocality is fundamental; it is rather a conjecture for the most cosmologically significant part of the quantum gravitational effective action. The underlying idea [28] is that the problem of the cosmological constant [29, 30] may have no resolution: general relativity really does have a large, positive cosmological constant, and this is what started primordial inflation. However, accelerated expansion led to the production of a vast ensemble of infrared gravitons [31], and the self-gravitation between these gravitons grew without bound as more and more of them came into causal contact. This self-gravitation provides a sort of quantum gravitational friction which slows inflation by an amount that eventually becomes nonperturbatively large. No one has yet devised a way of passing beyond perturbation theory to derive the result but the natural supposition is that this 
quantum gravitational effect eventually screened the large bare cosmological constant and brought inflation to a close.

Because what is being cancelled is a constant, whereas the screening mechanism is dynamical, depending on how many gravitons can see one another as the past light-cone opens up, it is obvious that the persistence of perfect screening can only occur in one geometry. We believe this "perfect" geometry is radiation domination, and that the transition to matter domination disrupts perfect screening, after which a small fraction of the original large bare cosmological constant peeks out from under the blanket of infrared gravitons which had previously completely screened it.

The key nonlocal ingredient in our model is the inverse scalar d'Alembertian $\mathbf{\square}^{-1}$ which can be roughly motivated [32,33] by the secular growth factors that arise in explicit loop corrections to gravitational radiation [34,35] and to gravitational forces [36, 37] on de Sitter background. For the rest, the Ricci scalar is the simplest curvature scalar upon which it might act, and the combination in $Y[g](x)$ seems to be the simplest form which both matches the perturbative secular growth on de Sitter and also changes sign inside gravitationally bound systems. At this stage there is of course no way to derive the nonlocal distortion function $f(Y)$, but simply accepting the model as a residual effect from the gravitational screening of inflationary gravitons does motivate two of its features which would otherwise seem oracular:

- There is an initial value surface upon which the initial conditions of $\square^{-1}$ can be defined; and

- There are modifications of gravity on large, but not small, distances without fine tuning or an explicit $\Lambda$.

\section{Enforcing the $\Lambda$ CDM Expansion History}

In this section, we solve for the distortion function $f(Y)$ which supports the $\Lambda \mathrm{CDM}$ expansion history without dark energy. We begin by specializing the model to cosmology, then describe the procedure for numerically determining the required $f(Y)$. The section closes with a very simple and accurate exponential fit to this function. 


\subsection{The Cosmological Sector}

Cosmology's geometry is well described by a scale factor $a(t)$,

$$
d s^{2}=-d t^{2}+a^{2}(t) d \vec{x} \cdot d \vec{x}
$$

whose expansion is quantified by the Hubble and first slow roll parameters,

$$
H \equiv \frac{\dot{a}}{a} \quad, \quad \epsilon \equiv-\frac{\dot{H}}{H^{2}}
$$

In this geometry the nonzero covariant derivative operators become,

$$
\square \longrightarrow-\left(\frac{d}{d t}\right)^{2}-3 H \frac{d}{d t}, D_{0} D_{0} \longrightarrow\left(\frac{d}{d t}\right)^{2}, D_{i} D_{j} \longrightarrow g_{i j} H \frac{d}{d t} .
$$

The time-time component of the gravitational field equations (8) is,

$$
3 H\left(\frac{d}{d t}+H\right)(1+U+f(Y))+\frac{1}{2}\left(\dot{X} \dot{U}+\dot{Y} \dot{V}+\dot{X}^{2}\right)=8 \pi G \rho
$$

where $\rho$ is the energy density without dark energy. The space-space component is $g_{i j}$ times,

$$
-\left(\frac{d^{2}}{d t^{2}}+2 H \frac{d}{d t}+2 \dot{H}+3 H^{2}\right)(1+U+f(Y))+\frac{1}{2}\left(\dot{X} \dot{U}+\dot{Y} \dot{V}+\dot{X}^{2}\right)=8 \pi G p
$$

where $p$ is the pressure, also without dark energy.

The best time variable is $N \equiv \ln \left(\frac{a_{0}}{a}\right)$, the number of e-foldings until the present. The various differentials and derivatives then simplify,

$$
d N=-H d t \quad, \quad \frac{d}{d t}=-H \frac{d}{d N} \quad, \quad \frac{d^{2}}{d t^{2}}=H^{2}\left(\frac{d^{2}}{d N^{2}}+\epsilon \frac{d}{d N}\right) .
$$

We seek to determine the function $f(Y)$ to enforce the $\Lambda$ CDM expansion history without a cosmological constant. This means the Hubble parameter, energy density and pressure take the forms,

$$
\begin{aligned}
H^{2} & =H_{0}^{2}\left(\Omega_{r} e^{4 N}+\Omega_{m} e^{3 N}+\Omega_{\Lambda}\right) \equiv H_{0}^{2} \times \widetilde{H}^{2}, \\
8 \pi G \rho & =3 H_{0}^{2}\left(\Omega_{r} e^{4 N}+\Omega_{m} e^{3 N}\right) \equiv H_{0}^{2} \times \widetilde{\rho}, \\
8 \pi G p & =3 H_{0}^{2} \times \frac{1}{3} \Omega_{r} e^{4 N} \equiv H_{0}^{2} \times \widetilde{p}
\end{aligned}
$$


where $H_{0}$ is the current value of the Hubble parameter and $\Omega_{r}, \Omega_{m}$ and $\Omega_{\Lambda}=1-\Omega_{r}-\Omega_{m}$ are the $\Lambda$ CDM fractions of the energy density in radiation, matter and vacuum energy 3 In this notation the scalar equations are,

$$
\begin{gathered}
{\left[\widetilde{H} e^{-3 N} X^{\prime}\right]^{\prime}=-12\left(1-\frac{1}{2} \epsilon\right) \widetilde{H} e^{-3 N}, \quad\left[\widetilde{H} e^{-3 N} Y^{\prime}\right]^{\prime}=\widetilde{H} e^{-3 N} X^{\prime 2},} \\
{\left[\widetilde{H} e^{-3 N} V^{\prime}\right]^{\prime}=-12\left(1-\frac{1}{2} \epsilon\right) \widetilde{H} e^{-3 N} f^{\prime}(Y) \quad, \quad U^{\prime}=-2 X^{\prime} V}
\end{gathered}
$$

where a prime denotes differentiation with respect to the natural argument - $Y$ for $f(Y)$ and $N$ for $\widetilde{H}(N), X(N), Y(N), U(N)$ and $V(N)$. Note that equations (23) give explicit integral expressions for $X^{\prime}(N)$ and $Y^{\prime}(N)$,

$$
\begin{aligned}
X^{\prime}(N) & =\frac{e^{3 N}}{\widetilde{H}(N)} \int_{N}^{\infty} d N^{\prime} \frac{e^{-3 N^{\prime}}}{\widetilde{H}\left(N^{\prime}\right)}\left[3 \Omega_{m} e^{3 N^{\prime}}+12 \Omega_{\Lambda}\right], \\
Y^{\prime}(N) & =-\frac{e^{3 N}}{\widetilde{H}(N)} \int_{N}^{\infty} d N^{\prime} e^{-3 N^{\prime}} \widetilde{H}\left(N^{\prime}\right)\left[X^{\prime}\left(N^{\prime}\right)\right]^{2} .
\end{aligned}
$$

Figure 1 shows $X(N)$ and $Y(N)$ and their derivatives.
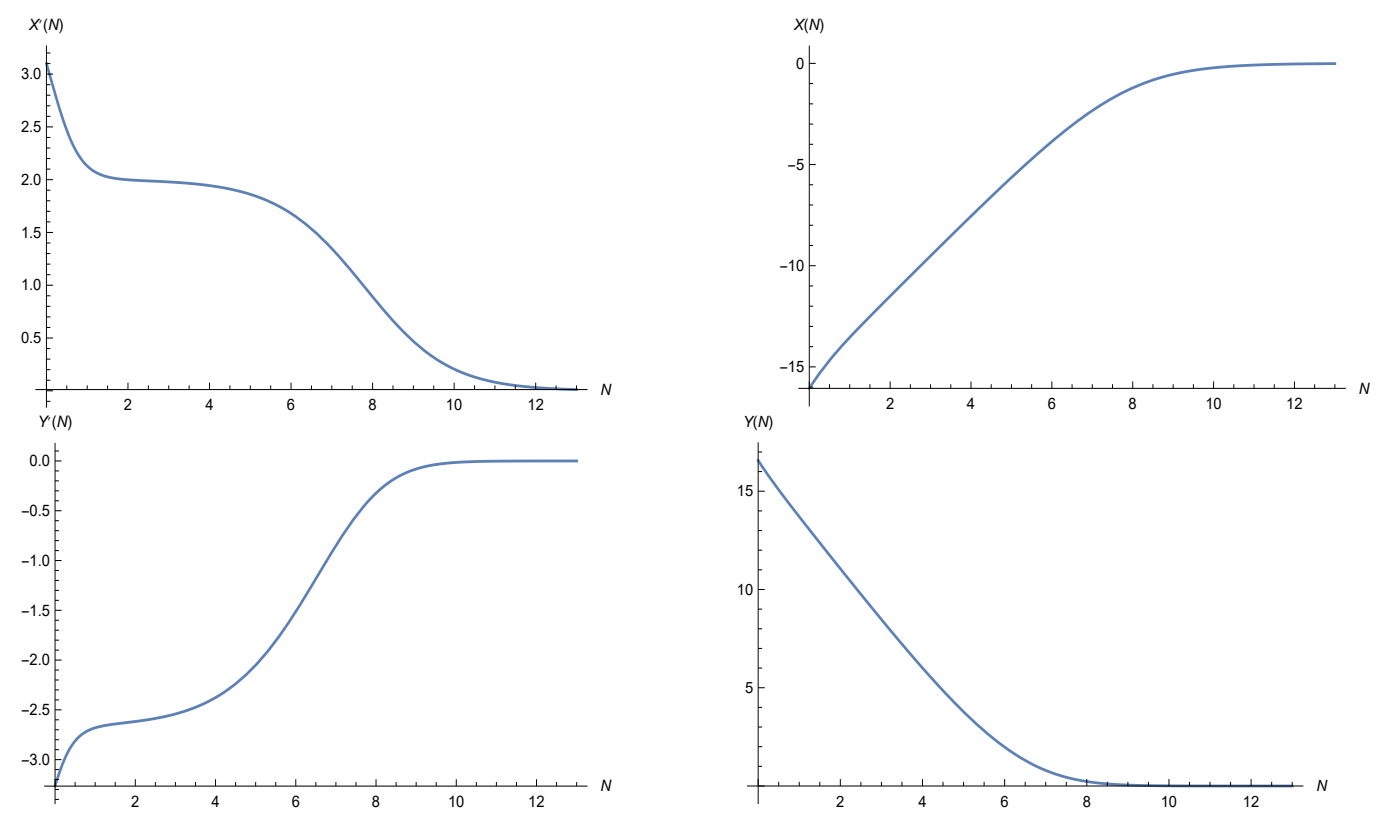

Figure 1: The left hand graphs show numerical simulations of $X^{\prime}(N)$ and $Y^{\prime}(N)$ as defined by (25-26). The right hand graphs give their integrals.

\footnotetext{
${ }^{3}$ We use $\Omega_{m} / \Omega_{r} \equiv 1+z_{e q} \simeq 3403, \Omega_{m} \simeq 0.3153$ and $\Omega_{\Lambda} \simeq 0.6847$ [38].
} 
Since $X^{\prime}(N)$ and $Y^{\prime}(N)$ have definite signs here, both $X$ and $Y$ are monotonic, hence invertible.

\subsection{The Reconstruction Procedure}

The two gravitational field equations are,

$$
\begin{aligned}
-3\left(\partial_{N}-1\right)[U+f(Y)]+\frac{1}{2}\left[X^{\prime} U^{\prime}+Y^{\prime} V^{\prime}+X^{\prime 2}\right] & =-\frac{3 \Omega_{\Lambda}}{\widetilde{H}^{2}} \\
-\left(\partial_{N}^{2}-(2-\epsilon) \partial_{N}+3-2 \epsilon\right)[U+f(Y)]+\frac{1}{2}\left[X^{\prime} U^{\prime}+Y^{\prime} V^{\prime}+X^{\prime 2}\right] & =\frac{3 \Omega_{\Lambda}}{\widetilde{H}^{2}} .
\end{aligned}
$$

As for the original model [2], the first step in constructing a nonlocal distortion function which supports the $\Lambda \mathrm{CDM}$ expansion history is to take the difference of (27) and (28),

$$
\left(\partial_{N}-3+\epsilon\right)\left(\partial_{N}-2\right)[U+f(Y)]=-\frac{6 \Omega_{\Lambda}}{\widetilde{H}^{2}} .
$$

This can be integrated to give exactly the same result as for the original model [2],

$$
U+f(Y)=-6 \Omega_{\Lambda} e^{2 N} \int_{N}^{\infty} d N^{\prime} \frac{e^{N^{\prime}}}{\widetilde{H}\left(N^{\prime}\right)} \int_{N^{\prime}}^{\infty} d N^{\prime \prime} \frac{e^{-3 N^{\prime \prime}}}{\widetilde{H}\left(N^{\prime \prime}\right)} \equiv g(N) .
$$

The next step is to derive a differential equation for the function,

$$
G(N) \equiv \frac{Y^{\prime}(N)}{X^{\prime}(N)} f^{\prime}(Y(N))-\frac{g^{\prime}(N)}{X^{\prime}(N)} .
$$

Differentiating relation (30), using (24) and dividing by $X^{\prime}(N)$ gives,

$$
-2 V(N)+G(N)=0 .
$$

Acting $\partial_{N}^{2}-(3-\epsilon) \partial_{N}$ on (32) and using relation (24) produces,

$$
\left(\partial_{N}-3+\epsilon\right) \partial_{N} G+24\left(1-\frac{1}{2} \epsilon\right) \frac{X^{\prime}}{Y^{\prime}} G=-24\left(1-\frac{1}{2} \epsilon\right) \frac{g^{\prime}}{Y^{\prime}} .
$$

The procedure from this point is clear: we numerically solve (33) for $G(N)$, extract $\partial_{N} f(Y)=Y^{\prime} \times f^{\prime}(Y)$ using relation (31), numerically integrate to recover $f(Y)$ as a function of $N$, and finally exploit the one-to-one relation between $Y$ and $N$ to numerically express $f(Y)$ as a function of $Y$. 


\subsection{Solution for $f(Y)$}

The initial conditions at large $N$ follow from exact results, derived in Appendix A, by retaining only the leading dependence on $\Omega_{\Lambda}$. Because $\Omega_{\Lambda}$ is irrelevant until late times, expressions (47-55) are accurate to three digits for $N>2$. The functions we need for equation (33) can be usefully expanded in powers of the variable $y \equiv\left(1+z_{e q}\right) e^{-N}$,

$$
\begin{aligned}
\epsilon & \longrightarrow+2-\frac{1}{2} y+\frac{1}{2} y^{2}-\frac{1}{2} y^{3}+O\left(y^{4}\right) \\
X^{\prime} & \longrightarrow+\frac{3}{2} y-\frac{5}{4} y^{2}+\frac{35}{32} y^{3}-\frac{63}{64} y^{4}+O\left(y^{5}\right), \\
Y^{\prime} & \longrightarrow-\frac{3}{4} y^{2}+\frac{33}{32} y^{3}-\frac{367}{320} y^{4}+\frac{4577}{3840} y^{5}+O\left(y^{6}\right), \\
g^{\prime} & \longrightarrow \frac{\Omega_{\Lambda} \Omega_{r}^{3}}{\Omega_{r}^{4}}\left\{\frac{4}{5} y^{4}-\frac{11}{14} y^{5}+\frac{429}{560} y^{6}-\frac{142}{192} y^{7}+O\left(y^{8}\right)\right\} .
\end{aligned}
$$

Because $1+z_{e q} \simeq 3403 \simeq \exp [+8.132]$ is so large, these expansions are only accurate for $N>10$. Employing the expansions (34)37) allows us to factorize the large $N$ limiting form of the differential operator in equation (33),

$$
F_{1}(y) \frac{d}{d N}\left\{F_{2}(y) \frac{d}{d N}\left[F_{3}(y) G\right]\right\}=F_{4}(y),
$$

where the four factors are,

$$
\begin{aligned}
& F_{1}(y)=\frac{1}{y^{4}}\left[1+\frac{3}{8} y-\frac{13}{960} y^{2}-\frac{13}{4608} y^{3}+\ldots\right] \\
& F_{2}(y)=y^{7}\left[1-\frac{5}{4} y+\frac{1151}{960} y^{2}-\frac{6071}{5760} y^{3}+\ldots\right], \\
& F_{3}(y)=\frac{1}{y^{3}}\left[1+\frac{7}{8} y+\frac{47}{960} y^{2}+\frac{137}{23040} y^{3}+\ldots\right], \\
& F_{4}(y)=\frac{\Omega_{\Lambda} \Omega_{r}^{3}}{\Omega_{m}^{4}}\left\{\frac{32}{5} y^{3}-\frac{136}{35} y^{4}+\frac{3869}{1050} y^{5}-\frac{2587}{720} y^{6}+\ldots\right\} .
\end{aligned}
$$

Expression (38) is a second order differential equation and possesses two homogeneous solutions. However, only one of these falls off for large $N$,

$$
G_{\mathrm{h}}(N)=\frac{1}{F_{3}(y)}=y^{3}-\frac{7}{8} y^{4}+\frac{43}{60} y^{5}-\frac{85}{144} y^{6}+O\left(y^{7}\right) .
$$


The large $N$ limiting form of $G(N)$ can be inferred from (38),

$$
G(N) \longrightarrow \frac{\Omega_{\Lambda} \Omega_{r}^{3}}{\Omega_{m}^{4}}\left\{\frac{32}{35} G_{\mathrm{h}}(N) \ln (y)+\frac{5}{14} y^{4}-\frac{1247}{4200} y^{5}+\frac{71117}{302400} y^{6}+O\left(y^{7}\right)\right\}
$$

This provides the initial conditions to evolve (33) from finite $N$.

The expansion (44) fixes the small $Y$ behavior of $f(Y)$,

$$
f(Y)=\frac{\Omega_{\Lambda} \Omega_{r}^{3}}{\Omega_{m}^{4}}\left\{-\frac{128}{105} Y^{2} \ln (Y)+O\left(Y^{2}\right)\right\} .
$$

Numerical evolution gives the full result, which is depicted in Fig. 2.
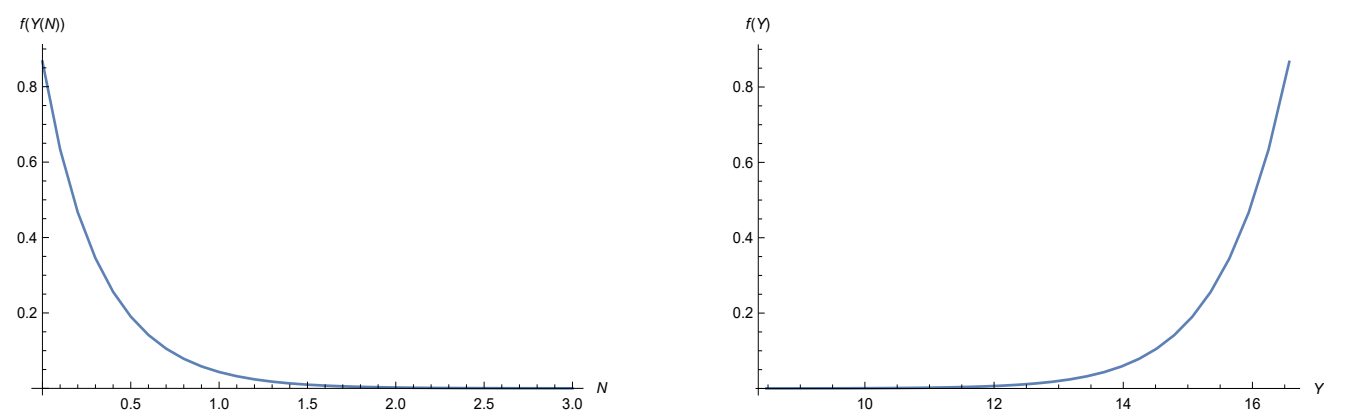

Figure 2: The left hand graph shows a numerical simulation of $f(Y)$ as a function of the evolution variable $N$. The right hand graph also gives $f(Y)$, but now as a function of its natural argument $Y$.
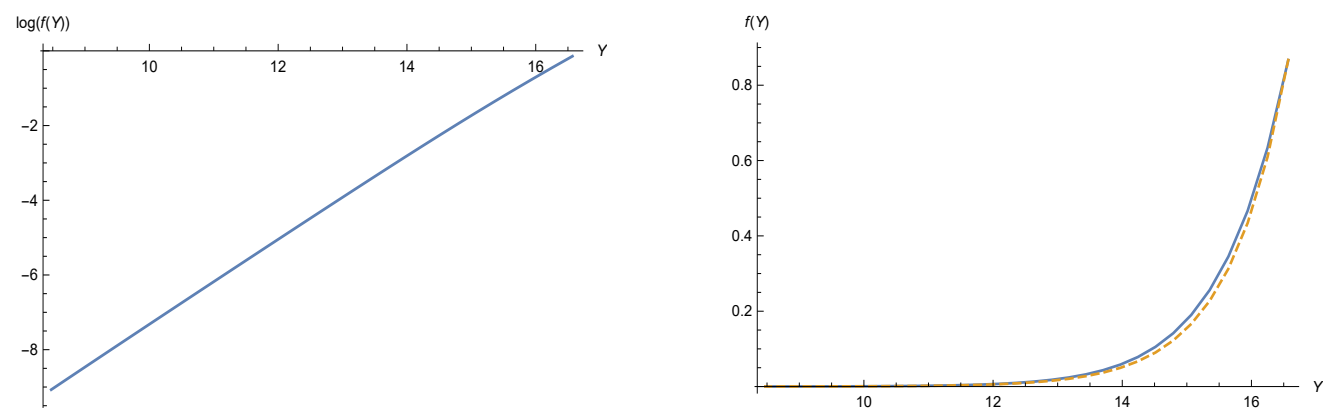

Figure 3: The left hand graph shows that $\ln [f(Y)]$ is nearly a straight line. The right hand graph compares the full numerical determination of $f(Y)$ (in solid, blue) to the resulting exponential fit (46) (in dashed, yellow). 
Figure 3 shows that $f(Y)$ is well fit by the strikingly simple form,

$$
f(Y) \simeq \exp [1.1(Y-16.7)] .
$$

\section{Discussion}

We have presented a simple variant of our original model [1 to explain the current phase of cosmic acceleration without dark energy. Like its ancestor, the new model is based on augmenting the Hilbert action by the addition of $R$ times a function of a dimensionless, nonlocal scalar; only the scalar has changed from $X[g]=\mathbf{\square}^{-1} R$ to $Y[g]=\mathbf{\square}^{-1} g^{\mu \nu} \partial_{\mu} X \partial_{\nu} X$. Both $X[g]$ and $Y[g]$ are quiescent during radiation domination, and thereafter only grow logarithmically, which provides a natural explanation for why the onset of acceleration is delayed to late in cosmic history. Both scalars also vanish for gravitational radiation which means that they do not affect the - tightly constrained - propagation velocity [25].

Fig. 4 shows that $Y$ is close to $-X$ for cosmology.
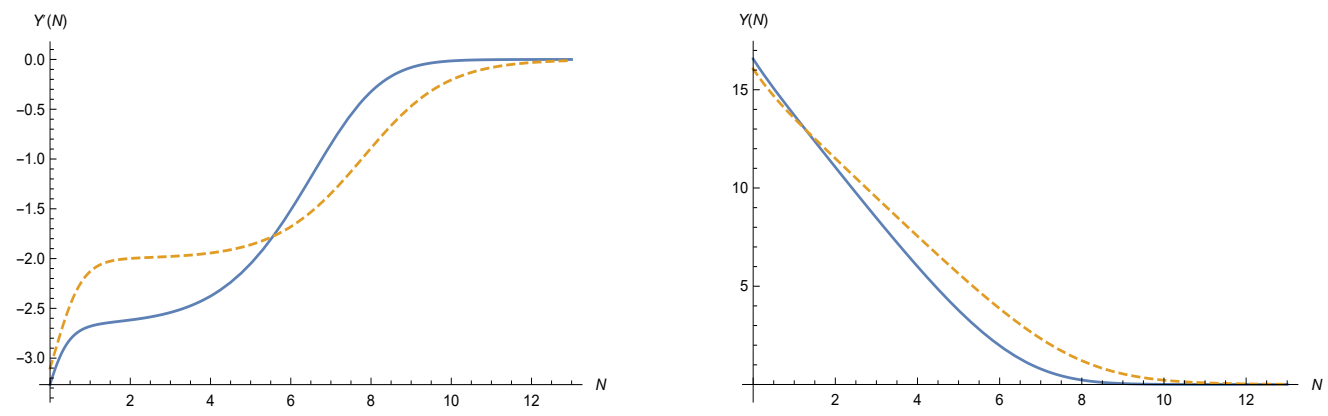

Figure 4: The left hand graph compares $Y^{\prime}(N)$ (solid blue) with $-X^{\prime}(N)$ (dashed yellow). The right hand graph compares $Y(N)$ (solid blue) with $-X(N)$ (dashed yellow).

This made it simple to determine the nonlocal distortion function $f(Y)$ numerically in order to reproduce exactly the $\Lambda$ CDM expansion history without dark energy. That was done in section 3, with results shown on Fig. 2, An unexpected consequence was the simple exponential approximation (46) for $f(Y)$, whose accuracy can be seen from Fig. 3 .

The new model differs from the original one in that $Y$ (unlike $X$ ) changes sign from cosmology (with $Y>0$ ) to gravitationally bound systems (with 
$Y<0)$. Because cosmology only fixes $f(Y)$ for $Y \geq 0$, with $f(0)=0$, simply assuming $f(Y)=0$ for $Y<0$ protects the model from changing the heavily constrained physics of gravitationally bound systems. The huge advantages of this model can be seen by comparison with $F(R)$ theories of gravity, which must invoke even more exotic physics such as the chameleon mechanism [39] to evade solar system constraints. Note also that the only stable choice of $F(R)$ which exactly reproduces the $\Lambda$ CDM expansion history is $F(R)=R-2 \Lambda$ [0].

Now that the nonlocal distortion function $f(Y)$ has been fixed the model is complete. Because $f(Y)$ has been chosen to exactly reproduce the $\Lambda \mathrm{CDM}$ expansion history, with no changes inside gravitationally bound systems, tests of the model must come from its predictions for cosmological perturbations and the growth of structures. Stability is another important constraint to study.

Finally, we return to the presumed local sources of our model, the gravitons of primordial inflation. Their loop effects can grow non-perturbatively strongly during the primordial inflation era [35], and $\mathbf{\square}^{-1}$ does correctly capture this growth on de Sitter background, but it is clearly a major unsolved problem to follow their temporal effects in any detail. We have no explanation, other than simplicity and dimensionlessness, for the combination $X=\square^{-1} R$, nor can we justify the appearance of $Y$, although it might be worth noting that nonlocal realizations of MOdified Newtonian Dynamics (MOND) [41-43] involve a similar nonlocal scalar [44,45]. The hope is that there is a master effective action describing the full range of cosmic history from the build-up of gravitational back-reaction during inflation, and giving rise to both the present model and to MOND as residual effects.

For now we can strictly only offer our phenomenological (but dimensionless) construction. Nevertheless, the presumed inflationary origin does provide two vital answers that otherwise seem unnatural: the existence of an initial value surface from which one may launch the initial conditions defining our inverse differential operators, i.e., the propagators, and why the corrections only modify classical general relativity on cosmological, rather than on the smaller (bound matter) scales, where no "improvement" is needed!

\section{Acknowledgements}

We are grateful to M. Maggiore for calling our attention to the problem with the original model. SD's work was supported by the U.S. Department 
of Energy, Office of Science, Office of High Energy Physics, under Award Number de-sc0011632. RPW was partially supported by NSF grants PHY1506513 and PHY-1806218, and by the Institute for Fundamental Theory at the University of Florida.

\section{Appendix: Exact Expressions to Leading Order in $\Omega_{\Lambda}$}

Expressions for $X(N), Y(N)$ and $g(N)$ simplify dramatically for large $N$ when $\Omega_{\Lambda}$ becomes negligible relative to $\Omega_{m} e^{3 N}$ and $\Omega_{r} e^{4 N}$. Setting $\Omega_{\Lambda}=0$ and making the change of variable $y \equiv \frac{\Omega_{m}}{\Omega_{r}} e^{-N}$ in expression (25) reduces $X^{\prime}(N)$ to an elementary function,

$$
X^{\prime}(N) \longrightarrow \frac{3}{y \sqrt{1+y}} \int_{0}^{y} \frac{y^{\prime} d y^{\prime}}{\sqrt{1+y^{\prime}}}=\frac{2(z-1)(z+2)}{z(z+1)}
$$

where $z \equiv \sqrt{1+y}$. Integrating expression (47) gives,

$$
X(N) \longrightarrow-2\left(\frac{z-1}{z+1}\right)-4 \ln \left(\frac{1}{2} z+\frac{1}{2}\right) .
$$

Setting $\Omega_{\Lambda}=0$ in expression (26) similarly reduces $Y^{\prime}(N)$ to an elementary function,

$$
\begin{aligned}
Y^{\prime}(N) & \longrightarrow \frac{-1}{y \sqrt{1+y}} \int_{0}^{y} d y^{\prime} \sqrt{1+y^{\prime}}\left[X^{\prime}\left(N^{\prime}\right)\right]^{2} \\
& =\frac{-8}{z(z+1)}\left[\frac{1}{3}\left(z^{2}+z-11\right)+\frac{2}{z+1}+\frac{4 \ln \left(\frac{1}{2} z+\frac{1}{2}\right)}{z-1}\right] .
\end{aligned}
$$

Integrating (50) to get $Y(N)$ produces a dilogarithm in addition to elementary functions,

$$
\begin{aligned}
Y(N) \longrightarrow \frac{8}{(z+1)^{2}}-\frac{112}{3(z+1)}+ & {\left[\frac{16}{3}-\frac{32 z}{z^{2}-1}\right] \ln \left(\frac{1}{2} z+\frac{1}{2}\right)+\frac{37}{24} } \\
& +8 \ln ^{2}\left(\frac{1}{2} z+\frac{1}{2}\right)+16 \operatorname{Li}_{2}\left(\frac{1}{2}-\frac{1}{2} z\right),
\end{aligned}
$$

where

$$
\operatorname{Li}_{2}(x) \equiv-\int_{0}^{x} d t \frac{\ln (1-t)}{t}
$$


The function $g(N)$ actually vanishes with $\Omega_{\Lambda}$ so its large $N$ limit derives from preserving the initial factor in (30),

$$
\begin{aligned}
g(N) & \longrightarrow-\frac{6 \Omega_{\Lambda} \Omega_{r}^{3}}{\Omega_{m}^{4} y^{2}} \int_{0}^{y} \frac{d y^{\prime}}{\sqrt{1+y^{\prime}}} \int_{0}^{y^{\prime}} d y^{\prime \prime} \frac{y^{\prime \prime 4}}{\sqrt{1+y^{\prime \prime}}} \\
& =-\frac{\Omega_{\Lambda} \Omega_{r}^{3}}{\Omega_{m}^{4}} \frac{(z-1)^{4}}{105}\left[28 z^{2}+112 z+156+\frac{64}{z+1}+\frac{32}{(z+1)^{2}}\right] .
\end{aligned}
$$

Differentiating this gives,

$$
g^{\prime}(N) \longrightarrow \frac{\Omega_{\Lambda} \Omega_{r}^{3}}{\Omega_{m}^{4}} \frac{(z-1)^{4}}{105}\left[84 z^{2}+336 z+508+\frac{352}{z}+\frac{96}{z(z+1)}+\frac{96}{z(z+1)^{2}}\right]
$$

All of these expressions are accurate to three digits for $N>2$.

\section{References}

[1] S. Deser and R. P. Woodard, Phys. Rev. Lett. 99, 111301 (2007) doi:10.1103/PhysRevLett.99.111301 [arXiv:0706.2151 [astro-ph]].

[2] C. Deffayet and R. P. Woodard, JCAP 0908, 023 (2009) doi:10.1088/1475-7516/2009/08/023 [arXiv:0904.0961 [gr-qc]].

[3] S. Deser and R. P. Woodard, JCAP 1311, 036 (2013) doi:10.1088/14757516/2013/11/036 arXiv:1307.6639 [astro-ph.CO]].

[4] T. M. C. Abbott et al. [DES Collaboration], arXiv:1810.02499 [astroph.CO].

[5] R. P. Woodard, Found. Phys. 44, 213 (2014) doi:10.1007/s10701-0149780-6 arXiv:1401.0254 [astro-ph.CO]].

[6] R. P. Woodard, Universe 4, no. 8, $88 \quad$ (2018) doi:10.3390/universe4080088 [arXiv:1807.01791 [gr-qc]].

[7] S. Park and S. Dodelson, Phys. Rev. D 87, no. 2, 024003 (2013) doi:10.1103/PhysRevD.87.024003 [arXiv:1209.0836 [astro-ph.CO]].

[8] S. Dodelson and S. Park, Phys. Rev. D 90, 043535 (2014) Erratum: [Phys. Rev. D 98, no. 2, 029904 (2018)] doi:10.1103/PhysRevD.90.043535, 10.1103/PhysRevD.98.029904 arXiv:1310.4329 [astro-ph.CO]]. 
[9] S. Park and A. Shafieloo, Phys. Rev. D 95, no. 6, 064061 (2017) doi:10.1103/PhysRevD.95.064061 [arXiv:1608.02541 [astro-ph.CO]].

[10] H. Nersisyan, A. F. Cid and L. Amendola, JCAP 1704, no. 04, 046 (2017) doi:10.1088/1475-7516/2017/04/046 arXiv:1701.00434 [astroph.CO]].

[11] S. Park, Phys. Rev. D 97, no. 4, 044006 (2018) doi:10.1103/PhysRevD.97.044006 [arXiv:1711.08759 [gr-qc]].

[12] L. Amendola, Y. Dirian, H. Nersisyan and S. Park, arXiv:1901.07832 [astro-ph.CO].

[13] T. Koivisto, Phys. Rev. D 77, 123513 (2008) doi:10.1103/PhysRevD.77.123513 arXiv:0803.3399 [gr-qc]].

[14] T. S. Koivisto, Phys. Rev. D 78, 123505 (2008) doi:10.1103/PhysRevD.78.123505 arXiv:0807.3778 [gr-qc]].

[15] Y. Z. Chu and S. Park, arXiv:1811.04647 [gr-qc].

[16] M. Maggiore and M. Mancarella, Phys. Rev. D 90, no. 2, 023005 (2014) doi:10.1103/PhysRevD.90.023005 [arXiv:1402.0448 [hep-th]].

[17] M. Maggiore, Fundam. Theor. Phys. 187, 221 (2017) doi:10.1007/9783-319-51700-1_16 [arXiv:1606.08784 [hep-th]].

[18] Y. Dirian, S. Foffa, N. Khosravi, M. Kunz and M. Maggiore, JCAP 1406, 033 (2014) doi:10.1088/1475-7516/2014/06/033 arXiv:1403.6068 [astro-ph.CO]].

[19] A. Barreira, B. Li, W. A. Hellwing, C. M. Baugh and S. Pascoli, JCAP 1409, no. 09, 031 (2014) doi:10.1088/1475-7516/2014/09/031 arXiv:1408.1084 [astro-ph.CO]].

[20] Y. Dirian and E. Mitsou, JCAP 1410, no. 10, 065 (2014) doi:10.1088/1475-7516/2014/10/065 arXiv:1408.5058 [gr-qc]].

[21] Y. Dirian, S. Foffa, M. Kunz, M. Maggiore and V. Pettorino, JCAP 1504, no. 04, 044 (2015) doi:10.1088/1475-7516/2015/04/044 arXiv:1411.7692 [astro-ph.CO]]. 
[22] Y. Dirian, S. Foffa, M. Kunz, M. Maggiore and V. Pettorino, JCAP 1605, no. 05, 068 (2016) doi:10.1088/1475-7516/2016/05/068 arXiv:1602.03558 [astro-ph.CO]].

[23] H. Nersisyan, Y. Akrami, L. Amendola, T. S. Koivisto and J. Rubio, Phys. Rev. D 94, no. 4, 043531 (2016) doi:10.1103/PhysRevD.94.043531 arXiv:1606.04349 [gr-qc]].

[24] E. Belgacem, A. Finke, A. Frassino and M. Maggiore, arXiv:1812.11181 [gr-qc].

[25] S. Boran, S. Desai, E. O. Kahya and R. P. Woodard, Phys. Rev. D 97, no. 4, 041501 (2018) doi:10.1103/PhysRevD.97.041501 arXiv:1710.06168 [astro-ph.HE]].

[26] S. Nojiri and S. D. Odintsov, Phys. Lett. B 659, 821 (2008) doi:10.1016/j.physletb.2007.12.001 [arXiv:0708.0924 [hep-th]].

[27] S. Park and R. P. Woodard, Phys. Rev. D 99, no. 2, 024014 (2019) doi:10.1103/PhysRevD.99.024014 [arXiv:1809.06841 [gr-qc]].

[28] N. C. Tsamis and R. P. Woodard, Nucl. Phys. B 474, 235 (1996) doi:10.1016/0550-3213(96)00246-5 hep-ph/9602315.

[29] S. Weinberg, Rev. Mod. Phys. 61, 1 (1989). doi:10.1103/RevModPhys.61.1

[30] S. M. Carroll, Living Rev. Rel. 4, 1 (2001) doi:10.12942/lrr-2001-1 astro-ph/0004075].

[31] L. P. Grishchuk, Sov. Phys. JETP 40, 409 (1975) [Zh. Eksp. Teor. Fiz. 67, 825 (1974)].

[32] N. C. Tsamis and R. P. Woodard, Annals Phys. 267, 145 (1998) doi:10.1006/aphy.1998.5816 [hep-ph/9712331].

[33] M. G. Romania, N. C. Tsamis and R. P. Woodard, Lect. Notes Phys. 863, 375 (2013) doi:10.1007/978-3-642-33036-0_13 [arXiv:1204.6558 [gr$\mathrm{qc}]]$.

[34] N. C. Tsamis and R. P. Woodard, Phys. Rev. D 54, 2621 (1996) doi:10.1103/PhysRevD.54.2621 hep-ph/9602317. 
[35] P. J. Mora, N. C. Tsamis and R. P. Woodard, JCAP 1310, 018 (2013) doi:10.1088/1475-7516/2013/10/018 arXiv:1307.1422 [gr-qc]].

[36] S. Park and R. P. Woodard, Phys. Rev. D 83, 084049 (2011) doi:10.1103/PhysRevD.83.084049 arXiv:1101.5804 [gr-qc]].

[37] S. Park, T. Prokopec and R. P. Woodard, JHEP 1601, 074 (2016) doi:10.1007/JHEP01(2016)074 arXiv:1510.03352 [gr-qc]].

[38] N. Aghanim et al. [Planck Collaboration], arXiv:1807.06209 [astroph.CO].

[39] J. Khoury and A. Weltman, Phys. Rev. Lett. 93, 171104 (2004) doi:10.1103/PhysRevLett.93.171104 astro-ph/0309300.

[40] P. K. S. Dunsby, E. Elizalde, R. Goswami, S. Odintsov and D. S. Gomez, Phys. Rev. D 82, 023519 (2010) doi:10.1103/PhysRevD.82.023519 arXiv:1005.2205 [gr-qc]].

[41] M. Milgrom, Astrophys. J. 270, 365 (1983). doi:10.1086/161130

[42] M. Milgrom, Astrophys. J. 270, 371 (1983). doi:10.1086/161131

[43] M. Milgrom, Astrophys. J. 270, 384 (1983). doi:10.1086/161132

[44] C. Deffayet, G. Esposito-Farese and R. P. Woodard, Phys. Rev. D 84, 124054 (2011) doi:10.1103/PhysRevD.84.124054 arXiv:1106.4984 [grqc]].

[45] C. Deffayet, G. Esposito-Farese and R. P. Woodard, Phys. Rev. D 90, no. 6, 064038 (2014) Addendum: [Phys. Rev. D 90, no. 8, 089901 (2014)] doi:10.1103/PhysRevD.90.089901, 10.1103/PhysRevD.90.064038 [arXiv:1405.0393 [astro-ph.CO]]. 